\title{
Three-dimensional visualization of cleared human pancreas cancer reveals that sustained epithelial-to-mesenchymal transition is not required for venous invasion
}

\author{
Seung-Mo Hong $\mathbb{1}^{1,2} \cdot$ DongJun Jung ${ }^{1,3} \cdot$ Ashley Kiemen ${ }^{4} \cdot$ Matthias M. Gaida ${ }^{5,6} \cdot$ Tadashi Yoshizawa $^{1}$ • \\ Alicia M. Braxton ${ }^{1}$ Michaël Noë $\mathbb{O}^{7} \cdot$ Gemma Lionheart $^{1} \cdot$ Kiyoko Oshima $^{1} \cdot$ Elizabeth D. Thompson $^{1,7}$. \\ Richard Burkhart ${ }^{8} \cdot$ Pei-Hsun Wu $\mathbb{1}^{4} \cdot$ Denis Wirtz ${ }^{1,4} \cdot$ Ralph H. Hruban $^{1,7} \cdot$ Laura D. Wood $^{1,7}$
}

Received: 26 July 2019 / Revised: 23 October 2019 / Accepted: 23 October 2019 / Published online: 7 November 2019

(c) The Author(s), under exclusive licence to United States \& Canadian Academy of Pathology 2019

\begin{abstract}
Venous invasion is three times more common in pancreatic cancer than it is in other major cancers of the gastrointestinal tract, and venous invasion may explain why pancreatic cancer is so deadly. To characterize the patterns of venous invasion in pancreatic cancer, 52 thick slabs (up to $5 \mathrm{~mm}$ ) of tissue were harvested from 52 surgically resected human ductal adenocarcinomas, cleared with a modified iDISCO method, and labeled with fluorescent-conjugated antibodies to cytokeratin 19, desmin, CD31, p53 and/or e-cadherin. Labeled three-dimensional (3D) pancreas cancer tissues were visualized with confocal laser scanning or light sheet microscopy. Multiple foci of venous and even arterial invasion were visualized. Venous invasion was detected more often in 3D (88\%, 30/34 cases) than in conventional 2D slide evaluation (75\%, 25/34 cases, $P<0.001)$. 3D visualization revealed pancreatic cancer cells crossing the walls of veins at multiple points, often at points where preexisting capillary structures bridge the blood vessels. The neoplastic cells often retained a ductal morphology (cohesive cells forming tubes) as they progressed from a stromal to intravenous location. Although immunolabeling with antibodies to e-cadherin revealed focal loss of expression at the leading edges of the cancers, the neoplastic cells within veins expressed e-cadherin and formed well-oriented glands. We conclude that venous invasion is almost universal in pancreatic cancer, suggesting that even surgically resectable PDAC has access to the venous spaces and thus the ability to disseminate widely. Furthermore, we observe that sustained epithelial-mesenchymal transition is not required for venous invasion in pancreatic cancer.
\end{abstract}

Supplementary information The online version of this article (https:// doi.org/10.1038/s41379-019-0409-3) contains supplementary material, which is available to authorized users.

Ralph H. Hruban

rhruban@jhmi.edu

$\triangle$ Laura D. Wood

ldwood@jhmi.edu

1 Department of Pathology, the Sol Goldman Pancreatic Cancer Research Center, Johns Hopkins Medical Institutions, Baltimore, MD, USA

2 Department of Pathology, Asan Medical Center, University of Ulsan College of Medicine, Seoul, Republic of Korea

3 Department of Medical Science, Graduate School, University of Ulsan, Seoul, Republic of Korea

\section{Introduction}

Pancreatic ductal adenocarcinoma (PDAC) is one of the leading causes of the cancer death in the United States. It is estimated that about 56,770 Americans will be diagnosed

4 Department of Chemical and Biomolecular Engineering, Johns Hopkins University, Baltimore, MD, USA

5 Institute of Pathology, University Hospital Heidelberg, Heidelberg, Germany

6 Institute of Pathology, University Medical Center Mainz, Mainz, Germany

7 Department of Oncology, the Sol Goldman Pancreatic Cancer Research Center, Johns Hopkins Medical Institutions, Baltimore, MD, USA

8 Department of Surgery, the Sol Goldman Pancreatic Cancer Research Center, Johns Hopkins Medical Institutions, Baltimore, MD, USA 
and 45,750 patients will die of PDAC in 2019 [1]. Most patients with pancreatic cancer are diagnosed with advanced disease, usually liver metastases, and only $10 \%$ of patients have localized disease [1]. Even patients with low-stage disease who undergo surgery only have a $34 \% 5$ yearsurvival rate [1]. Although the aggressiveness of PDAC is clearly due to several factors, it has been hypothesized that the high prevalence venous invasion in PDAC explains the rapid development of liver metastases and therefore the aggressiveness of disease in most patients with PDAC [2].

The reported prevalence $(65 \%)$ of venous invasion is higher in PDAC than in cancers of other abdominal organs $[3,4]$. Because veins in the pancreas drain directly into the liver, venous invasion in PDAC may explain the almost universal development of liver metastases in patients with this disease [2-6]. Venous invasion in PDACs is not only very common, but it also has a distinctive morphology. The neoplastic cells within the veins replace the endothelial cells and grow as well-oriented lumen-forming cells along the inner wall of the vessels, creating a unique pancreatic intraepithelial neoplasia-like histology [3]. Our understanding of the process of venous invasion of PDAC beyond this is limited in part due to emphasis on thin twodimensional (2D) hematoxylin and eosin (H\&E) tissue sections [7]. Therefore, the goal of the present study is to characterize venous invasion in human PDAC in 3D.

Technical advances in tissue clearing, antibody penetration, and advanced microscopes now enable the 3D visualization of dense fibrotic tissues, including human PDACs [7, 8]. We previously reported the 3D analysis of human PDAC using clearing and a single marker (cytokeratin 19) [8]. We reported that infiltrating cancer cells preferentially grow in the connective tissue parallel to blood vessels, and we also observed neoplastic cells growing within blood vessels [8]. In order to more precisely define the process of venous invasion in PDAC, we now apply multiple markers (antibodies to cytokeratin 19, desmin, p53, CD31, and e-cadherin) to a larger wellcharacterized series of cleared surgically resected PDACs. We confirm that venous invasion occurs in the vast majority of PDACs and observe that sustained epithelial-mesenchymal transition (EMT) is not required for venous invasion.

\section{Materials and methods}

\section{Patients and tissue preparation}

After approval from the institutional review boards, a total of 52 pancreas tissue slabs (up to $20 \times 10 \times 5 \mathrm{~mm}^{3}$ ) were examined. These included 34 fresh cancer tissues harvested from 34 patients who underwent pancreatic resection at the Johns Hopkins Medical Institutions for PDAC, and 18 pancreas tissue slabs from formalin-fixed paraffin embedded (FFPE) PDAC samples from 18 patients who underwent surgical resection at the University Hospital Heidelberg, Heidelberg, Germany. These latter cancers were selected because they were known to have venous invasion by 2D H\&E microscopy.

\section{Fresh samples}

All fresh pancreatic tissues were processed as previously described [8]. In brief, tissues were fixed overnight at room temperature in $80 \%$ methanol $/ 20 \%$ dimethyl sulfoxide (DMSO) to dehydrate the tissues and precipitate proteins to prevent autolysis by pancreatic enzymes, and then the tissues were rehydrated and fixed in $4 \%$ paraformaldehyde at room temperature for 1 day. They were then dehydrated with serially concentrated methanol $(1 \times 50 \%$ methanol, $1 \times$ $80 \%$ methanol, $1 \times 90 \%$ methanol, and $3 \times 100 \%$ methanol), chilled at $4{ }^{\circ} \mathrm{C}$ for $1 \mathrm{~h}$ in $100 \%$ methanol, and incubated overnight in $66 \%$ dichloromethane $/ 33 \%$ methanol at room temperature. To oxidize endogenous pigments and autofluorescent proteins, tissues were washed twice in $100 \%$ methanol and then $5 \% \mathrm{H}_{2} \mathrm{O}_{2}$ was added for overnight incubation. Tissues were rehydrated in $1 \mathrm{x}$ phosphatebuffered saline (PBS) and then washed twice for $1 \mathrm{~h}$ each in PBS/0.2\% Triton X-100 (Millipore Sigma, St. Louis, MO). Next, the tissues were incubated for 2 days in a permeabilization solution of $\mathrm{PBS} / 20 \% \mathrm{DMSO} / 0.2 \%$ Triton $\mathrm{X}-100 / 0.3 \mathrm{~mol} / \mathrm{L}$ glycine at $37^{\circ} \mathrm{C}$. Blocking reagent of $6 \%$ donkey serum, $10 \%$ DMSO in PTx.2 was incubated for 2 days.

\section{Formalin-fixed, paraffin-embedded samples}

Eighteen FFPE tissues were released from the paraffin block by dissolving the paraffin in xylene at $37^{\circ} \mathrm{C}$. The tissues then were washed three times in $100 \%$ methanol and chilled at $4{ }^{\circ} \mathrm{C}$ for $1 \mathrm{~h}$, followed by overnight incubation in $66 \%$ dichloromethane $/ 33 \%$ methanol at room temperature. The tissues were then washed twice in $100 \%$ methanol, and $5 \%$ $\mathrm{H}_{2} \mathrm{O}_{2}$ was added to the $100 \%$ methanol for overnight incubation. Tissues were rehydrated in PBS and washed twice for an hour each in PBS/0.2\% Triton X-100, and then incubated in PBS/20\% DMSO/0.2\% Triton X-100/0.3 mol/L glycine at $37^{\circ} \mathrm{C}$ for 2 days. Glycine was added to prevent background labeling.

\section{Multiplex immunolabeling}

Nonspecific antigens were blocked by incubating the tissues in PBS/0.2\% Triton X-100/10\% DMSO/6\% donkey serum at $37^{\circ} \mathrm{C}$ for 2 days. Samples then were washed twice in PBS $/ 0.2 \%$ Tween- 20 with $10 \mathrm{mg} / \mathrm{mL}$ heparin each at $37{ }^{\circ} \mathrm{C}$ for $1 \mathrm{~h}$. Heparin was added to prevent background immunolabeling. Multiple primary antibodies, including 
cytokeratin 19 (EP1580Y, rabbit monoclonal; final dilution, 1:200; Abcam, Cambridge, UK), desmin (goat polyclonal; final concentration, 1:100, LifeSpan Biosciences, Seattle WA), CD31 (JC/70A, mouse monoclonal; final concentration, 1:100, Thermo Fisher Scientific, Waltham, MA), p53 (DO-7, mouse monoclonal; final concentration, 1:100, Thermo Fisher Scientific), and e-cadherin (M168, mouse monoclonal; final concentration, 1:150; Abcam) were used. These antibodies were applied in varying combinations, with a maximum of four antibodies used on any one tissue. Tissue from all 34 freshly harvested PDAC cases were labeled with at least both cytokeratin 19 and desmin. In addition, 24 of the 34 freshly harvested PDAC cases were also labeled with antibodies to p53, 24 PDAC cases with antibodies to CD31 and 8 with antibodies to e-cadherin. Diffuse nuclear p53 labeling was noted in 14 of the 24 cases (58\%), which aided in the identification of invasive carcinoma. Similarly, all 18 FFPE PDAC cases were labeled with at least both cytokeratin 19 and desmin. In addition, 7 of these 18 PDACs were also labeled with antibodies to CD31 and 8 with antibodies to e-cadherin.

Because dense desmoplastic stroma in PDACs is a significant physical barrier for the penetration of antibodies, four approaches were used to increase antibody penetration: First, the antibody concentration was gradually increased for 4 consecutive days. Second, during these 4 days of antibody incubation, the tissues were centrifuged consecutively for $12 \mathrm{~h}$ at $600 \times g$ and shaken at $37^{\circ} \mathrm{C}$ for $12 \mathrm{~h}$. After the primary antibody was applied to the tissues, they were washed five times with PBS $/ 0.2 \%$ Tween- 20 with $10 \mathrm{mg} / \mathrm{mL}$ heparin for $1 \mathrm{~h}$ each at room temperature. Third, pepsin-digested secondary antibody fragments with smaller molecular weights than intact $\operatorname{IgG}$ antibodies were used to increase tissue penetration of the secondary antibodies. The fragmented secondary antibodies, including Alexa Fluor 488-conjugated AffiniPure $\mathrm{F}\left(\mathrm{ab}^{\prime}\right)_{2}$ fragment donkey antirabbit IgG (Jackson ImmunoResearch,West Grove, PA), DyLight 405-conjugated AffiniPure $\mathrm{F}\left(\mathrm{ab}^{\prime}\right)_{2}$ fragment donkey anti-goat IgG (Jackson ImmunoResearch), and Cyanine 3-conjugated AffiniPure $\mathrm{F}\left(\mathrm{ab}^{\prime}\right)_{2}$ fragment, donkey antimouse IgG (Jackson ImmunoResearch), were incubated for 4 days and protected from light. During this time, the tissues were alternatively centrifuged for $12 \mathrm{~h}$ at $600 \times g$ and shaken for $12 \mathrm{~h}$ both at $37^{\circ} \mathrm{C}$. Fourth, after centrifugation and shaking, the tissues were sonicated for $1 \mathrm{~h}$ at $37^{\circ} \mathrm{C}$. The tissues were then washed five times with PBS/0.2\% Tween20 with $10 \mathrm{mg} / \mathrm{mL}$ heparin for $1 \mathrm{~h}$ each at room temperature and protected from light exposure.

\section{Tissue clearing}

After the immunolabeling, the tissues were dehydrated with serially concentrated methanol $(70 \%$ methanol, $95 \%$ methanol, and $3 \times 100 \%$ methanol), incubated with $66 \%$ dichloromethane $/ 33 \%$ methanol for $3 \mathrm{~h}$, and $100 \%$ dichloromethane for $15 \mathrm{~min}$ twice, and were finally transferred to dibenzyl ether (DBE) overnight.

\section{Tissue imaging}

Immunolabeled tissues were visualized in 3D primarily with a confocal laser scanning microscope (LSM800; Carl Zeiss, Jena, Germany) and when lower power views were needed with a Light Sheet Microscope (Ultramicroscope II; LaVision BioTec, Bielefeld, Germany). The Ultramicroscope II is equipped with a Neo sCMOS camera (Andor Technology, Belfast, UK) and a $\times 4$ objective lens that was immersed in DBE in the imaging chamber.

Alexa 488 signals of CK19 expressing epithelial cells (normal ductal and cancer cells) were visualized with a bandpass filter set with an excitation range of $480 / 40 \mathrm{~nm}$ and an emission range of 525/50 nm. DyLight 405 signals of desmin-expressing cells (smooth muscle cells) were visualized with a filter set with an excitation range of 400/ $40 \mathrm{~nm}$ and an emission range of $421 / 50 \mathrm{~nm}$. Cyanine $3 \mathrm{sig}$ nals of CD31expressing endothelial cells, of e-cadherin labeled cells, or p53 labeled cancer cells were visualized using a filter set with an excitation range of $550 / 40 \mathrm{~nm}$ and an emission range of $570 / 50 \mathrm{~nm}$. Although autofluorescence of the tissues was reduced as described in the "Methods" section, the elastic lamina and collagen of blood vessels still had detectable autofluorescence. This autofluorescence, combined with the unique morphology of elastic lamina, was used to identify vessels in addition to desmin labeling. Autofluorescence was observed in an additional filter set of the LaVision Ultramicroscope II (excitation, $405 / 40 \mathrm{~nm}$; emission, $460 / 50 \mathrm{~nm}$ ).

For the LSM800 microscope, the tissue was submerged in DBE in a glass bottom cell culture dish to match the refractive index and to obtain a flat surface at the interface between materials of different refractive indices. We used the $\times 5, \times 10$, and $\times 20$ objectives. Consecutive $2 \mathrm{D}$ images with $\mathrm{Z}$ stacks were visualized with Zen (Ver. 2.3, Zeiss) and 3D images were reconstructed with IMARIS software (Ver. 9.4, Bitplane, Zurich Switzerland).

\section{Validation}

Seven cases were embedded in paraffin after the tissues had been cleared and visualized. These seven cases were then sectioned, stained with H\&E, and the findings in the cleared tissues were compared to the $\mathrm{H} \& \mathrm{E}$ findings.

Briefly, DBE was removed by washing with serially diluted methanol, and the tissues were rehydrated with PBS. Tissues then were FFPE, and sectioned for routine H\&E staining. The pathologies observed in the cleared tissues 
were then compared with those features in the H\&E stained slides. In five of the seven cases, the entire tissue block was serially sectioned and one slide taken every $13 \mu \mathrm{m}$. These serial slides were stained with $H \& E$ and then digitized (NanoZoomer-XR, Hamamatsu, Hamamatsu City, Japan). The scanned slides were then registered in $3 \mathrm{D}$ and areas with venous invasion were reconstructed. Serial H\&E sections were aligned to each other with rigid image registration using MATLAB 2019a. Registration between adjacent images was achieved by finding the rotational angle and translation which maximized cross correlation between images. This resulted in a continuous digital volume of H\&E sections. By manually annotating foci of venous invasion on these serial sections, 3D reconstructions of venous invasion were created and allowed $3 \mathrm{D} H \& \mathrm{H}$ validation of the observations made in the cleared tissues.

\section{Results}

\section{Patient and tumor characteristics}

Clinicopathologic characteristics of the 34 fresh PDAC cases included in this study are summarized in Supplementary Table 1 . Briefly, the mean age ( \pm standard deviation) of the patients was $69.7 \pm 11.2$ years. Male to female ratio was $1: 1$. The mean tumor size was $3.3 \pm 0.9 \mathrm{~cm}$ (range, $1.8-5.7 \mathrm{~cm})$. Twenty-three cases $(68 \%)$ were located in the pancreatic head, 18 (53\%) were moderately differentiated, and $25(74 \%)$ had vascular invasion of any type (lymphatic and or venous) present in the original 2D H\&E sections utilized for clinical diagnosis. Seven patients had received neoadjuvant therapy before resection.

Of the 18 FFPE PDACs, the mean age ( \pm standard deviation) of the patients was $58.1 \pm 10.9$ years. Male to female ratio was $2: 1$. The mean tumor size was $3.8 \pm 0.7 \mathrm{~cm}$ (range, $2.5-5.5 \mathrm{~cm}$ ). Fourteen cases $(78 \%)$ were located in the pancreatic head. All 18 FFPE cases were selected based on the presence of vascular invasion on the original diagnostic H\&E slides and so they were not considered in analyses of the prevalence of venous invasion. None of these 18 had received preoperative neoadjuvant therapy.

\section{Identifying muscular vessels in cleared tissues}

Blood vessels and ducts were identified based on desmin labeling and the distinct morphology of the ducts and of the arterial elastic lamina. Ducts were easily distinguished from vessels. Ducts are in the center of lobular units, while vessels are at the periphery [9]. As a result, normal interlobular ducts are surrounded by acinar cells. In contrast, vessels, because they are in the stroma at the periphery of lobules in the pancreas, are not surrounded by acinar cells

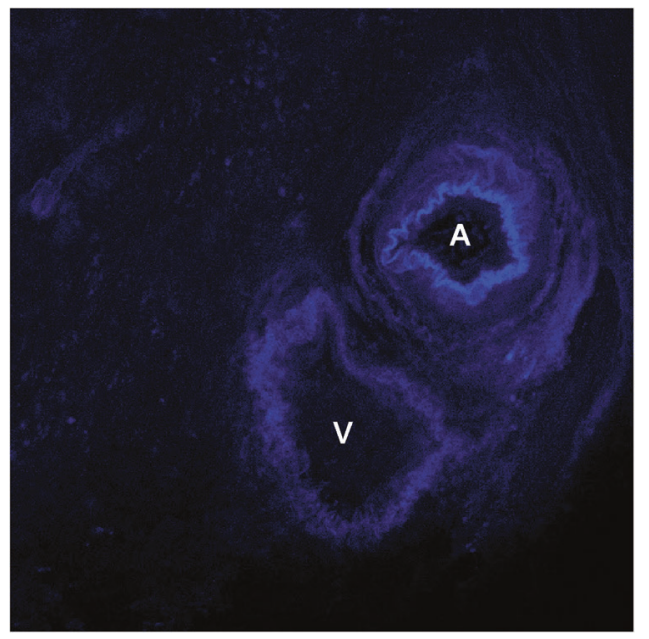

Fig. 1 The combination of morphology and patterns of immunolabeling can be used to define key structures in cleared tissues. Desmin immunolabeling of arterial walls (A) is more intense and the media of arteries is thicker than those of muscular veins (V). The concentric wavy autofluorescent lines of the elastic lamina also highlights arterial walls. [Blue labeling for desmin highlights the muscular layer in blood vessels]

[9]. In addition, desmin labeling of the media of blood vessels was distinguished from the weaker desmin labeling of the walls of pancreatic ducts. The media of veins is thicker and label more intensely than do the walls of ducts.

Veins and arteries were distinguished using several features. In general, desmin immunolabeling of arterial walls was more intense than the labeling of both ducts and veins, and the media of arteries was thicker than those of muscular veins. In addition, arterial walls contain elastic lamina, which was visualized as auto-fluorescing regularly concentric wavy lines (Fig. 1). The autofluorescence of vessels was easily distinguished from other sources of autofluorescence, including fibroblasts and their associated collagen. First, the nonspecific autofluorescence from fibroblasts provided weaker signals on different excitation wavelengths with different LASERs, including 405 and $488 \mathrm{~nm}$. Second, most muscular veins run parallel to an artery in the pancreas [9].

Cells of invasive carcinoma were easily distinguished from lobular collections of acinar cells. Unlike acinar cells, the cells of invasive carcinoma vary in size and form irregular haphazard 3D clusters or single cells.

\section{Comparison of detection of venous invasion between 2D and 3D analyses}

All 2D diagnostic H\&E slides were rereviewed from the 34 fresh cases, and the prevalence of venous invasion identified in the fresh cleared tissue was compared with the prevalence of venous invasion in the original $2 \mathrm{D}$ diagnostic $\mathrm{H} \& \mathrm{E}$ slides prepared before clearing. Thus, the venous invasion 
evaluated in the 2D diagnostic H\&E slides was from an adjacent but separate block of tumor. Venous invasion was detected more often in 3D $(88 \%, 30 / 34$ cases) than in conventional 2D H\&E slide evaluation $(74 \%, 25 / 34$ case; chi-square test, $P<0.001)$. The 18 FFPE cases were not included in these analyses, as they had been selected specifically because venous invasion was identified on diagnostic H\&E slides.

\section{D pathologic features of PDAC in the stroma adjacent to muscular blood vessels}

The neoplastic cells of invasive PDAC typically formed tubes/cords, larger globular masses, or single cells. As previously described using a single antibody, when cords of neoplastic cells approached vessels, they tracked parallel to the vessels (Fig. 2a, b, and Supplementary Movies 1 and 2) [8].

\section{D pathologic features of vascular invasion}

Venous invasion was identified in 30 of the 34 fresh cases. In 23 of these 30 cases we observed neoplastic cells crossing the media of the involved veins (Fig. 2c-f). Rather than individual cells crossing the media of veins, we most often observed ( 21 of 23 foci of venous invasion) cords and tube-like growths of cancer cells crossing the media of veins (Fig. 2c, d and Supplementary Movies 3 and 4). These cords and tubes often appeared intact, that is the cords were long and relatively uniform without disruption. This pattern was not universal, as in some instances small clusters and individual neoplastic cells were observed spanning the media of veins (Fig. 2e). When venous invasion was identified, the neoplastic cells frequently crossed the muscular venous wall at multiple points along the length of the vessel (Fig. 2f, Supplementary Movie 5). For example, in Fig. 2f and Supplementary Movie 5, at least three separate foci can be identified at which the neoplastic cells cross the media of the involved vessel. Some of the points at which neoplastic cells traversed the media of vessels were associated with the presence of endothelial cells (Fig. 2d and Supplementary Movie 4), raising the possibility that the neoplastic cells exploit local weaknesses in the vessel wall created by these preexisting capillary structures. Arterial invasion was much less common (seen in six cases), and when it occurred the pattern was usually that of individual cells or a few clusters of cells within arterial lumina (Fig. 2e). We did not observe tumor cells penetrating the media of muscular arteries.

The 3D morphologic features of vascular invasion in the 18 FFPE PDAC cases matched those observed in the 34 fresh PDAC cases.

\section{E-cadherin expression}

E-cadherin plays an important role in cell-to-cell adhesion, cell-to-cell recognition, and epithelial polarity, and loss of e-cadherin is considered to be one marker of EMT [10-14]. As expected, e-cadherin labeling was intact in neoplastic cells that formed well-oriented tubes and clusters (Supplementary Fig. 2 and Supplementary Movie 8). However, the patterns of e-cadherin expression as neoplastic cells approached, crossed and then populated the intraluminal portions of vessels was unexpected. We observed small tongues of cells projecting out from the leading edges of larger globular masses of neoplastic cells in the stroma (Fig. 3a). The neoplastic cells within the globular masses expressed e-cadherin, while these tongues of cells did not label. A similar pattern was observed as the neoplastic cells crossed the media of veins, with intact e-cadherin labeling in the large cohesive cluster of neoplastic cells and loss of ecadherin only in the tips of small tongues of cells extending out from these clusters (Fig. 3b). In contrast, the neoplastic cells entirely within the lumina of veins often had diffuse ecadherin labeling and formed tubes of well-oriented cells with well-defined lumina (Fig. 3c, d, Supplementary Fig. 1 and Supplementary Movies 6 and 7).

The combined findings of cell morphology, including cohesive cells forming well-oriented ductal structures, and intact e-cadherin expression suggest that although there may be transient EMT, the neoplastic cells rapidly reexpress markers of epithelial differentiation once inside the veins; thus sustained EMT is not required for venous invasion.

\section{H\&E validation}

After clearing, seven slabs of tissue with multiple foci of venous invasion in $3 \mathrm{D}$ were selected and processed for routine $\mathrm{H} \& \mathrm{E}$ microscopy. These sections revealed definite foci of vascular invasion in all seven representative cases, and these foci matched the foci of vascular invasion observed in the cleared 3D images. In addition, it was noted that foci of venous invasion were associated with mixed inflammatory cells, intraluminal fibrin thrombi, and disruption of endothelial cells [15].

Serial 2D H\&E slides from these seven cases were digitized and $3 \mathrm{D}$ reconstructions of the $\mathrm{H} \& \mathrm{E}$ sections were created. These 3D reconstructions revealed that the neoplastic cells extended within the veins for a distance (Fig. 4). The mean length of vascular invasion after reconstruction of $3 \mathrm{D} H \& E$ images was $565.7 \pm 502.3 \mu \mathrm{m}$ (median, $457.5 \mu \mathrm{m}$; range, 65-1800 $\mu \mathrm{m}$ ). The neoplastic cells even involved several branches of venous structures as these veins branched (Fig. 4). Both of these findings are consistent with observations made in the 3D immunofluorescently labeled cleared tissues. 

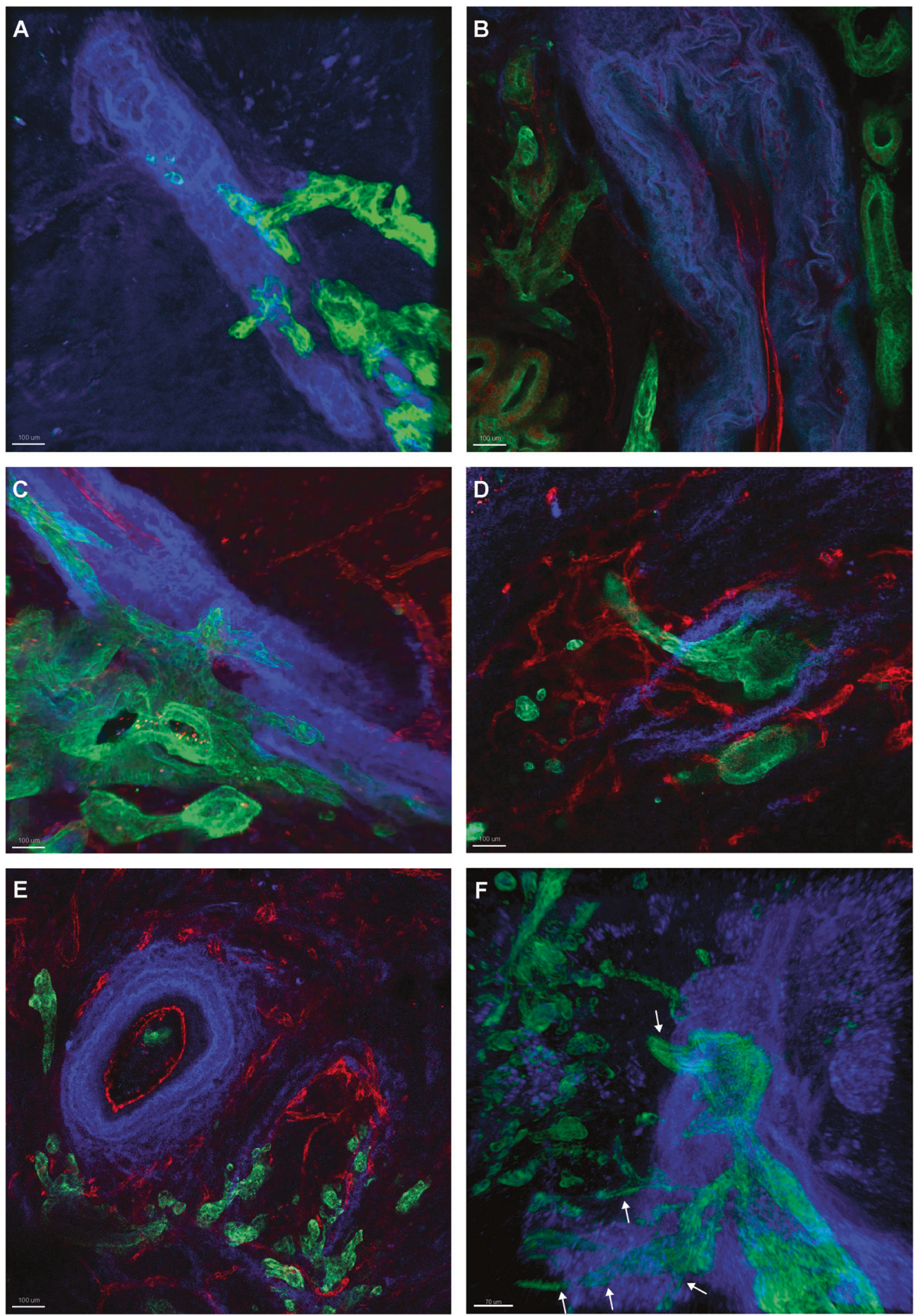

Fig. 2 Representative images of multicolor immunofluorescent labeling of pancreatic ductal adenocarcinoma tissues with venous invasion. a A cord of cancer cells approaches and then infiltrates a muscular blood vessel. b Arterial wall is surrounded by cancer cells. Some cancer cells show nuclear p53 labeling (orange nuclei, lower left). c Cohesive cancer cells infiltrate into a muscular vein. d Cancer cells from tubular structure inside of a vein and a well-formed tube of neoplastic cells crosses the media of the vein. e Small clusters of

cancer cells grow into a vein (lower right). Note the adjacent artery (upper left). f Cords of cancer cells form well-oriented tube within a vein, and cross the media of the vein at several points (arrows) along the vein. Focal cross reactivity of the antibody to desmin to islets of Langerhans is noted in this case. [Green labeling for cytokeratin 19 highlights the neoplastic cells, blue labeling for desmin highlights the muscular layer in blood vessels, red labeling in $\mathbf{b}-\mathbf{e}$ for CD31 marks endothelial cells, and nuclear red labeling for p53 in b] 


\section{Fig. 3 Epithelial-to-}

mesenchymal transition is not sustained in venous invasion. a Loss of e-cadherin expression (arrows) in small tongues of neoplastic cells projecting out from a larger globular mass of neoplastic cells with intact ecadherin expression. b Loss of e-cadherin expression (arrows) in small tongues of neoplastic cells projecting into the lumen of a vein. A detached cluster of neoplastic cells within the vein also show loss of e-cadherin labeling. c A well-formed tube of neoplastic cells with intact ecadherin labeling extending Well-oriented cancer cells form a long tube growing within a vein. Note the adjacent artery right of the vein. Focal cross reactivity of the antibody to desmin to islets of Langerhans is noted in this case. [Green labeling for cytokeratin 19, blue labeling for desmin, and red labeling in a-c for e-cadherin] within the lumen of a vein. $\mathbf{d}$ with wavy elastic lamina to the


B slides reveals branching of vascular invasion over a long distance. a Representative 2D $H \& E$ slide image of vascular invasion. Cancer cells within the veins replace the endothelial cells and form a well-oriented lumen. Yellow circle indicates outermost contour of cancer cells within vein, b 3D reconstruction of venous invasion (red). Yellow color matches location of the representative image shown in a venous invasion occurs in almost all pancreatic cancers ( $90 \%)$; (2) pancreatic cancer cells traverse the wall of veins at multiple point; (3) once in veins, the neoplastic cells often form well-oriented cohesive tubes of cells that replace the endothelial cells; [15] (4) the neoplastic cells extend for a distance (at least $1.8 \mathrm{~mm}$ ) within the veins; (5)
Here we report a detailed 3D study of the patterns of venous invasion in a large series of surgically resected pancreatic cancers using tissue clearing and immunolabeling with multiple antibodies. This 3D analysis revealed that: (1) 
sustained EMT is not required during process of vascular invasion.

Venous invasion is more common in PDAC than it is in cancers of other abdominal organs [2]. The previously reported prevalence of venous invasion in PDACs based on the evaluation of conventional $\mathrm{H} \& \mathrm{E}$ slide is $65 \%[3,4]$, as compared with $24 \%$ in gastric carcinomas and $21 \%$ in colon cancers [16-19]. Here, using cleared 3D samples we were able to identify venous invasion in close to $90 \%$ of pancreatic cancers that were of low enough stage to be surgically resected. This high prevalence in incompletely sampled cancers (a portion of each cancer underwent routine processing for clinical diagnosis) suggests that venous invasion, even in low-stage disease, is an almost universal process in human PDAC. This venous invasion may be one of the reasons for the rapid clinical progression of PDACs from stage I to stage IV disease [2, 20].

One of the advantages of studying tissues in $3 \mathrm{D}$ is that one can more accurately calculate the 3D sizes and behavior of lesions. In this study we observed pancreatic cancers growing for a mean of $565.7 \mu \mathrm{m}$ as they extended intraluminally within veins. This is remarkable as this represents a minimum number, as most of the foci of venous invasion extended to the edges of the tissue blocks. In addition, we observed that as they grew within veins, the neoplastic cells transgressed the walls of the veins at multiple points. Similarly, Nguyen et al., using in vitro models, reported that PDAC cells invade through matrix and into the lumina of vessels, where they ablate the endothelial cells, leaving behind tumor-filled luminal structures [15]. Our observations suggest that intravascular grow may also be responsible for local extension and for intra-pancreatic spreading of cancer cells. We also confirm our previous observation that invasive carcinoma of the pancreas often tracks parallel to veins within the perivascular connective tissue. The presence of a glandular structure next to a muscular blood vessel in the pancreas has been used clinically to aid in the diagnosis of pancreatic cancers in 2D H\&E stained slides [9].

Traditionally, EMT has been believed to play a critical role in dissemination of neoplastic cells from the primary tumor site and intravasation into blood vessels [21-23]. Ecadherin loss has been reported to be a critical part of EMT [24]. For example, Onder et al. observed that loss of ecadherin induced multiple downstream transcription factors, including Twist, and resulted in metastatic dissemination [11]. We observed intact e-cadherin labeling in cohesive neoplastic cells in the stromal and within the lumina of veins. This finding, coupled with the morphologic findings of cohesive cells forming tubes, indicates that while transient EMT occurs, sustained EMT is not required for venous invasion in human PDAC. Similarly, Brabletz et al. reported heterogeneous EMT at the invasive front of colorectal cancers [25]. They found that mesenchymal genes are expressed at the advancing edges of colorectal cancers, but the central portion of the tumors retain markers of the epithelial phenotype [25]. Our observation of heterogeneous e-cadherin labeling of small clusters of cancer cells at the leading edges of larger groups of cohesive neoplastic cells also parallels observations by Ligorio et al. recently reported in mouse models of pancreatic cancer [26]. They reported heterogeneity of RNA expression at the single cell level within individual cancer glands [26]. This focal loss of e-cadherin protein expression at the projecting ends of cancer cell clusters suggests a transient heterogeneity in the expression of the EMT phenotype which can be quickly reversed once the cells grow within veins.

We should acknowledge some limitations of the present study. For technical reasons, we could apply up to four antibodies at once in our 3D studies. As such, we were limited in the markers we employed to define EMT. While the combination of morphology (loss of cell cohesion and lack of formation of well-oriented glands) and loss of ecadherin expression was used to define EMT, the assessment of additional markers of EMT such as TWIST in future studies could help further solidify our conclusions [11]. In addition, assessment of expression of other proteins in 3D may also help elucidate the mechanisms underlying venous invasion in the pancreas. We should also note that we were able to evaluate only the cells that remained in the samples studied. We are not able to comment on the nature of the cells that gained access to the vasculature and then spread beyond the pancreas. Finally, although review by experienced pathologists revealed that neoplastic cells in the stroma, at the point of penetration of muscular veins, and within veins all showed intact e-cadherin labeling, there are currently no approaches to reliably quantify this labeling in cleared 3D pancreatic tissue specimens to make more detailed comparisons. Such comparisons will require development of new approaches for 3D image analysis.

In summary, 3D histopathologic features of venous invasion in human PDACs after tissue clearing demonstrate that venous invasion is present in close to $90 \%$ of pancreatic cancers. Pancreatic cancer cells cross the vascular media at multiple points along a single vessel, and sustained EMT is not required during process of vascular invasion. It is our hope that future studies will lead to the identification of novel approaches to block this process and prevent the rapid progression of disease.

Acknowledgements This work was presented in part at the 2019 annual meeting of United States and Canadian Academy of Pathology, National Harbor, MD. LW is supported by the Sol Goldman Pancreatic Cancer Research Center, Susan Wojcicki and Dennis Troper, the Michael Rolfe Foundation for Pancreatic Cancer Research, Buffone Family Gastrointestinal Cancer Research Fund, Kaya Tuncer Career Development Award in Gastrointestinal Cancer Prevention, AGA -Bernard Lee Schwartz Foundation Research Scholar Award in 
Pancreatic Cancer, Sidney Kimmel Foundation for Cancer Research Kimmel Scholar Award, AACR-Incyte Corporation Career Development Award for Pancreatic Cancer Research, Joseph C Monastra Foundation, The Gerald O Mann Charitable Foundation (Harriet and Allan Wulfstat, Trustees). MN is supported by The Nijbakker-Morra Foundation, The Lisa Waller Hayes Foundation. SMH is supported by the National Research Foundation of Korea (NRF) funded by the Ministry of Science, ICT, and Future Planning (NRF2019R1H1A2102016). MMG is supported by the German Research Foundation (Ga 1818/2-1). The authors thank Drs Michael Goggins and Anne Macgregor-Das for providing p53-positive pancreatic ductal adenocarcinomas, Drs Robert A. Anders, Alan K. Meeker, Christopher M. Heaphy, and Scot C. Guo for helpful discussions for this project, and Ms Yuan Kai and Ms Barbara Smith for their technical support.

\section{Compliance with ethical standards}

Conflict of interest The authors declare that they have no conflict of interest.

Publisher's note Springer Nature remains neutral with regard to jurisdictional claims in published maps and institutional affiliations.

\section{References}

1. Siegel RL, Miller KD, Jemal A. Cancer statistics, 2019. CA Cancer J Clin. 2019;69:7-34.

2. Hruban RH, Gaida MM, Thompson E, Hong SM, Noë M, Brosens LA, et al. Why is pancreatic cancer so deadly? The pathologist's view. J Pathol. 2019;248:131-41.

3. Hong SM, Goggins M, Wolfgang CL, Schulick RD, Edil BH, Cameron JL, et al. Vascular invasion in infiltrating ductal adenocarcinoma of the pancreas can mimic pancreatic intraepithelial neoplasia: a histopathologic study of 209 cases. Am J Surg Pathol. 2012;36:235-41.

4. Yamada M, Sugiura T, Okamura Y, Ito T, Yamamoto Y, Ashida $\mathrm{R}$, et al. Microscopic venous invasion in pancreatic cancer. Ann Surg Oncol. 2018;25:1043-51.

5. Suenaga M, Fujii T, Kanda M, Takami H, Okumura N, Inokawa $\mathrm{Y}$, et al. Pattern of first recurrent lesions in pancreatic cancer: hepatic relapse is associated with dismal prognosis and portal vein invasion. Hepatogastroenterology. 2014;61:1756-61.

6. Hamada Y, Nakayama Y. Aggressive venous invasion in the area of carcinoma correlates with liver metastasis as an index of metastasis for invasive ductal carcinoma of the pancreas. Pancreatology. 2017;17:951-5.

7. Hong SM, Noë M, Hruban CA, Thompson ED, Wood LD, Hruban RHA. "Clearer" view of pancreatic pathology: a review of tissue clearing and advanced microscopy techniques. Adv Anat Pathol. 2019;26:31-9.

8. Noë M, Rezaee N, Asrani K, Skaro M, Groot VP, Wu PH, et al. Immunolabeling of cleared human pancreata provides insights into three-dimensional pancreatic anatomy and pathology. Am J Pathol. 2018;188:1530-5.

9. Sharma S, Green KB. The pancreatic duct and its arteriovenous relationship: an underutilized aid in the diagnosis and distinction of pancreatic adenocarcinoma from pancreatic intraepithelial neoplasia. A study of 126 pancreatectomy specimens. Am J Surg Pathol. 2004;28:613-20.

10. Jeanes A, Gottardi CJ, Yap AS. Cadherins and cancer: how does cadherin dysfunction promote tumor progression? Oncogene. 2008;27:6920-9.

11. Onder TT, Gupta PB, Mani SA, Yang J, Lander ES, Weinberg RA. Loss of E-cadherin promotes metastasis via multiple downstream transcriptional pathways. Cancer Res. 2008;68:3645-54.

12. Cao D, Maitra A, Saavedra JA, Klimstra DS, Adsay NV, Hruban RH. Expression of novel markers of pancreatic ductal adenocarcinoma in pancreatic nonductal neoplasms: additional evidence of different genetic pathways. Mod Pathol. 2005;18:752-61.

13. Winter JM, Ting AH, Vilardell F, Gallmeier E, Baylin SB, Hruban $\mathrm{RH}$, et al. Absence of E-cadherin expression distinguishes noncohesive from cohesive pancreatic cancer. Clin Cancer Res. 2008;14:412-8.

14. Zeisberg M, Neilson EG. Biomarkers for epithelial-mesenchymal transitions. J Clin Investig. 2009;119:1429-37.

15. Nguyen DT, Lee E, Alimperti S, Norgard RJ, Wong A, Lee JJ, et al. A biomimetic pancreatic cancer on-chip reveals endothelial ablation via ALK7 signaling. Sci Adv. 2019;5:eaav6789.

16. Gresta LT, Rodrigues-Junior IA, de Castro LP, Cassali GD, Cabral MM. Assessment of vascular invasion in gastric cancer: a comparative study. World J Gastroenterol. 2013;19:3761-9.

17. Inada K, Shimokawa K, Ikeda T, Ozeki Y. The clinical significance of venous invasion in cancer of the stomach. Jpn J Surg. 1990;20:545-52.

18. Hwang C, Lee S, Kim A, Kim YG, Ahn SJ, Park DY. Venous invasion in colorectal cancer: impact of morphologic findings on detection rate. Cancer Res Treat. 2016;48:1222-8.

19. Betge J, Pollheimer MJ, Lindtner RA, Kornprat P, Schlemmer A, Rehak $\mathrm{P}$, et al. Intramural and extramural vascular invasion in colorectal cancer: prognostic significance and quality of pathology reporting. Cancer. 2012;118:628-38.

20. Yu J, Blackford AL, Dal Molin M, Wolfgang CL, Goggins M. Time to progression of pancreatic ductal adenocarcinoma from low-to-high tumour stages. Gut. 2015;64:1783-9.

21. Nieto MA, Huang RY, Jackson RA, Thiery JP. EMT: 2016. Cell. 2016;166:21-45.

22. Micalizzi DS, Haber DA, Maheswaran S. Cancer metastasis through the prism of epithelial-to-mesenchymal transition in circulating tumor cells. Mol Oncol. 2017;11:770-80.

23. Chen T, You Y, Jiang H, Wang ZZ. Epithelial-mesenchymal transition (EMT): a biological process in the development, stem cell differentiation, and tumorigenesis. J Cell Physiol. 2017;232:3261-72.

24. Serrano-Gomez SJ, Maziveyi M, Alahari SK. Regulation of epithelial-mesenchymal transition through epigenetic and posttranslational modifications. Mol Cancer. 2016;15:18.

25. Brabletz T, Jung A, Reu S, Porzner M, Hlubek F, Kunz-Schughart LA, et al. Variable beta-catenin expression in colorectal cancers indicates tumor progression driven by the tumor environment. Proc Natl Acad Sci USA. 2001;98:10356-61.

26. Ligorio M, Sil S, Malagon-Lopez J, Nieman LT, Misale S, Di Pilato M, et al. Stromal microenvironment shapes the intratumoral architecture of pancreatic. Cancer Cell. 2019;178:160-75 e27. 